\title{
Improved playback uniformity of random-phase free holograms by pixel separation method
}

\author{
Michał Makowski, ${ }^{* 1}$ Tomoyoshi Shimobaba ${ }^{2}$ \\ ${ }^{1}$ Faculty of Physics, Warsaw University of Technology, 75 Koszykowa, 00-662 Warsaw, Poland \\ ${ }^{2}$ Graduate School of Engineering, Chiba University, 1-33 Yayoi-cho, Inage-ku, Chiba 263-8522, Japan
}

Received October 11, 2021; accepted December 17, 2021; published December 31, 2021

\begin{abstract}
Random-phase free computer-generated holograms offer excellent quality of virtually noise-free playback of low-frequency images, but have limited efficiency in the case of highly contrast binary images with dominant high spatial frequencies. The introduction of weak random phase allows partial suppression of this problem, but causes strong noise in the outcome. Here we present the influence of a pixel separation technique on the uniformity of far field reconstructions from such random-phase free holograms. We show improved image quality with no additional speckle noise.
\end{abstract}

Most commonly used Computer-Generated Holograms $(\mathrm{CGH})$ are computed with a degree of randomized phase. This allows the utilization of a full spectrum of possible angles of diffraction after the hologram in order to fulfill intensity constraints in the output plane $[1,2]$. On the other hand, such an approach comprises inevitable random interferences of light rays forming adjacent points of the holographic image [3]. This problem can be partly solved by introducing a periodical void between image points by a pixel separation method [4].

Random-Phase Free (RPF) holograms have the unique property of correct image reconstruction at Fresnel distances without the introduction of randomized phase and consecutive noise [5]. Their disadvantage is poor rendering of high-contrast, binary images where high spatial frequencies are dominant. Although iterative error diffusion algorithms of their optimization were presented [6], their usage comprises a significant increase of the computational burden. A method of improving the uniformity of replayed intensity patterns from the RPF CGHs would be advantageous.

Here we present the application of a non-iterative technique of pixel separation used for the RPF CGHs. It assumes splitting a given input image into a set of subframes, each of which contains a subset of pixels. Usually the choice of pixels is random or periodical, but in every case all subframes combined (summed) give the exact copy of the input frame. The aim of eliminating a set of pixels in subframes is to create void spaces between image points reconstructed from the hologram. Such voids introduce zero amplitude spots in the image space and as

*E-mail: michal.makowski@pw.edu.pl such eliminate some of the uncontrolled interferences, thus contributing to the suppression of speckle noise, which was described in details [3]. Exemplary input image (USAF-1951 resolution test) used in this work and its four subframes are shown in Fig. 1.

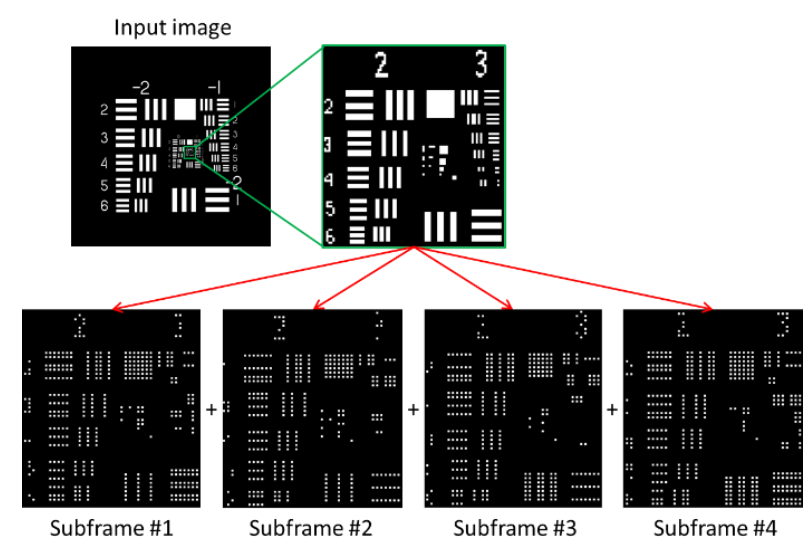

Fig. 1. Test input image for holographic computation and its subframes with pixel separation value $N=2$.

The distance between adjacent illuminated (i.e. non-void) pixels is called the separation constant $N$, here $N=2$. The total number of the subframes is equal to $N^{2}$, here 4.

In this section we will present the results of numerical simulations of holographic image reconstructions from CGHs computed with four methods. Method 1 assumes the computation of a RPF hologram from a non-pixel-separated input frame [5]. In this case no error diffusion or random phase inclusion was made. Methods 2 and 3 are modifications of Method 1 in which the input field is altered by adding a weak random phase in the range of $\pm \pi / 4$ and $\pm \pi / 2$, respectively. Method 4 is our proposed method, which uses pixel separation with $N=2$. The numerical reconstructions of the encoded USAF-1951 test image are gathered in Fig. 2. 
All images were computed with an angular spectrum propagation method $[7,8]$ on matrices of $2048^{2}$ complex samples on in-house software. The sampling was set at $8 \mu \mathrm{m}$ and the wavelength was fixed at $633 \mathrm{~nm}$. The propagation distance was $200 \mathrm{~mm}$, which did not violate the Nyquist limit and thus ensured that the maximal angles of diffraction enabled the light from all corners of the $\mathrm{CGH}$ to converge in the played back on-axis image.
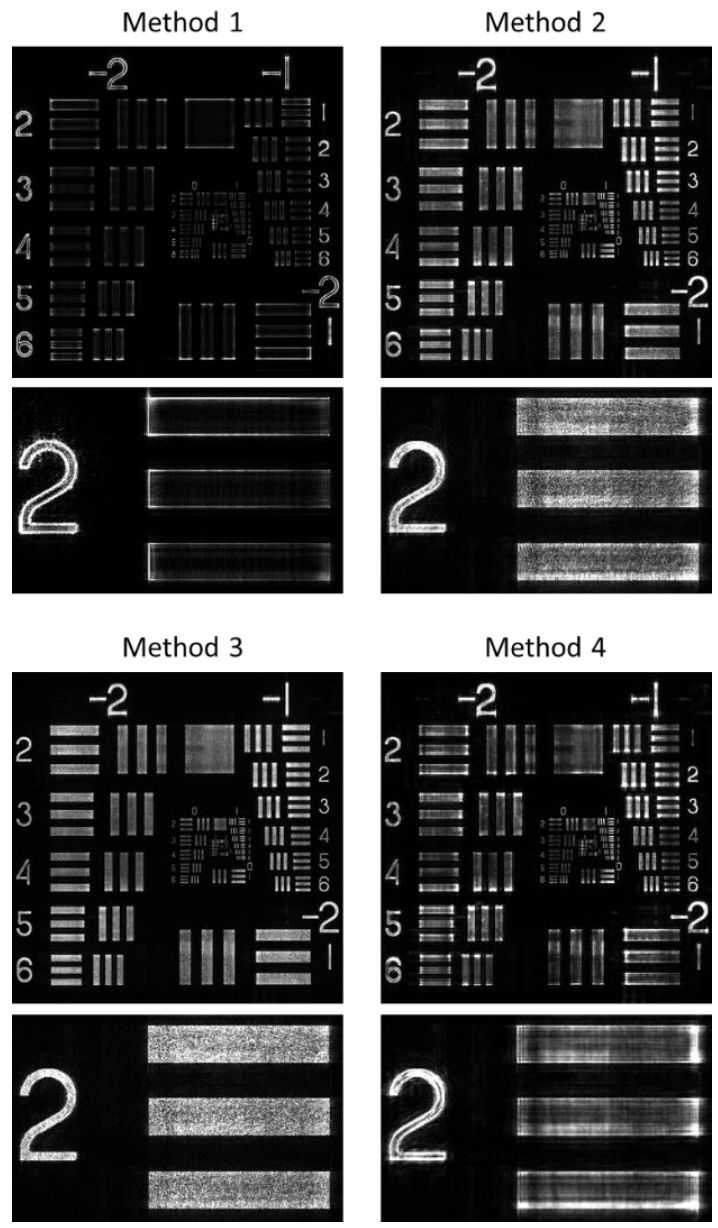

Fig. 2. Numerical holographic reconstruction of the test image with four compared methods. Close-ups of the $2^{\text {nd }}$ group are shown for noise

$$
\text { estimation. }
$$

From the qualitative analysis of Fig. 2 one can notice that Method 1 performs very poorly and requires serious upgrade. The previously presented methods of improving image uniformity based on the addition of random phase (Method 2 and 3) inevitably introduce grain-like noise, which stands in conflict with the main advantage of the RPF holograms, i.e. virtually no speckle noise. On the other hand, they significantly improve the uniformity of the insides of the bars seen in the USAF pattern. Method 4 seems to be a good compromise between the requirements to fill the insides of the objects and to keep the noise at a low level.

\begin{tabular}{|l|r|r|}
\cline { 2 - 3 } \multicolumn{1}{c|}{} & SSIM & noise contrast \\
\hline Method 1 & $18 \%$ & $53 \%$ \\
\hline Method 2 & $64 \%$ & $43 \%$ \\
\hline Method 3 & $70 \%$ & $44 \%$ \\
\hline Method 4 & $54 \%$ & $29 \%$ \\
\hline
\end{tabular}

Fig. 3. Statistics of image quality for the compared methods.

The quantitative analysis of the numerical results is given in Fig. 3. The proposed methods features the lowest noise level of all compared, while the structural similarity (SSIM) is on the middle level, which makes this stand in full agreement with subjective qualitative analysis.

The proposed method of pixel separation applied to RPF holograms has proven to be effective at little additional computational cost, mostly devoted to the calculation of three additional CGHs. The disadvantage of this method is experimental complexity which lies in the necessity of quickly displaying four sub-holograms on a spatial light modulator in order to get them integrated in the detector, e.g. human eye. Exemplary CGHs of subframes of the used USAF-1951 test image are shown in Fig. 4, while their numerical reconstructions showing fragmented sub-images are shown in Fig. 5.
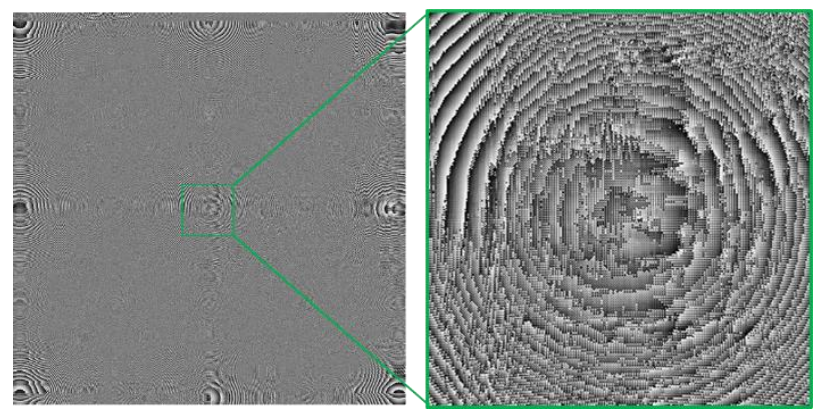

Fig. 4. Exemplary CGH of a pixel-separated subframe with its magnified central region. 

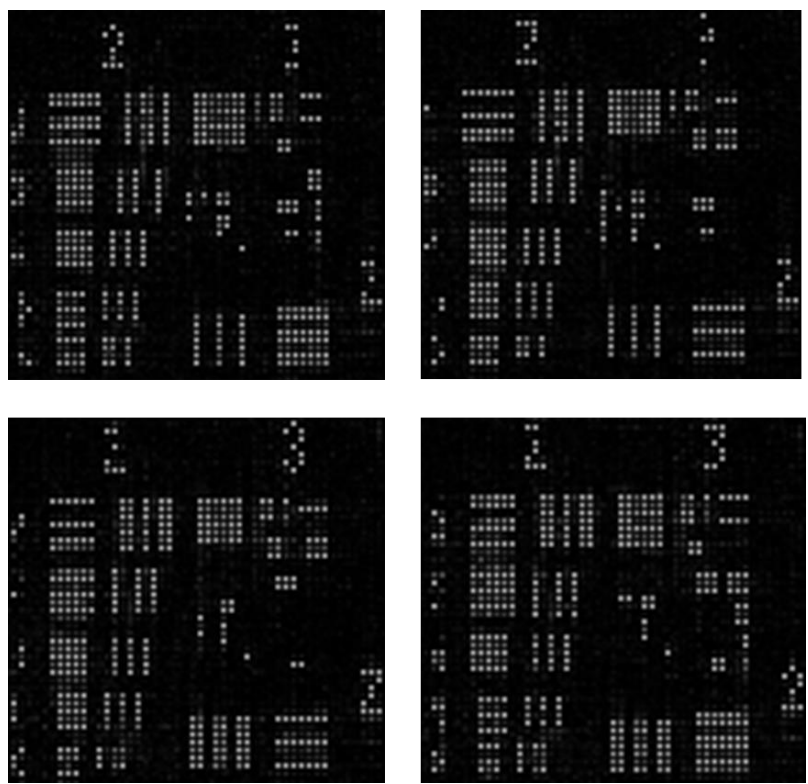

Fig. 5. Central magnifications of the intensity fields reconstructed from the four sub-holograms of the test image.

The experimental validation, as well as finding the optimal value of pixel separation distance and/or optimal pattern of inter-pixel voids will be the matter of our future research

\section{References}

[1] J.W. Goodman, Introduction to Fourier optics (Roberts and Company, 2005).

[2] R.W. Gerchberg, W.O. Saxton, Optik 35, 237 (1972).

[3] M. Makowski, Opt. Express 21, 29205 (2013).

[4] I. Ducin, T. Shimobaba, M. Makowski, K. Kakarenko, A. Kowalczyk, Jaroslaw Suszek, M. Bieda, A. Kolodziejczyk, M. Sypek, Opt. Comm. 340, 131 (2015).

[5] T. Shimobaba, T. Ito, Opt. Express 23, 9549 (2015)

[6] T. Shimobaba, T. Kakue, Y. Endo, R. Hirayama, D. Hiyama S. Hasegawa, Y. Nagahama, M. Sano, M. Oikawa, T. Sugie, T. Ito, Opt. Express 23, 17269 (2015).

[7] M. Sypek, Opt. Comm. 116, 43 (1995).

[8] K. Matsushima, T. Shimobaba, Opt. Express 17, 19662 (2009). 